\title{
Myocardial viability imaging using a novel non-breath-hold cardiac MRI protocol: a comparative assessment with ${ }^{18}$ F-FDG PET
}

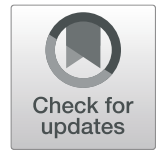

Rohit Aggarwal ${ }^{1 *}$ D, Geetika Singla ${ }^{2}$, Harkirat Singh ${ }^{3}$, Raju Augustine George ${ }^{2}$ and Indrani Mukhopadhyay ${ }^{4}$

\begin{abstract}
Background: Delayed contrast enhancement on cardiac magnetic resonance imaging is a well-established MRI technique for the evaluation of myocardial tissue viability. A comprehensive cardiac MRI protocol for myocardial viability comprises of multiple breath hold sequences to provide information regarding chamber volumes, myocardial mass, and function in addition to viability. However, its routine use is limited mainly by lengthy acquisition time and patient's inability to hold breath in multiple breath hold sequences. The important question to which the referring cardiologists/cardiac surgeons are seeking answer is that "What are the viable vascular territories that will benefit from revascularization?" In this study, we have analyzed the utility of non-breath-hold rapid delayed contrast-enhanced cardiac MR imaging protocol for myocardial viability assessment with 18 -flourodeoxyglucose positron emission tomography ( ${ }^{18} \mathrm{~F}$-FDG PET) as the reference standard.

Results: Forty patients of ischemic heart disease who met the inclusion criteria were included. All patients underwent both ${ }^{18} \mathrm{~F}$-FDG PET and delayed contrast-enhanced cardiac magnetic resonance imaging as per the non-breath-hold protocol. In this free-breathing protocol, the breath hold cine sequences were omitted and, after localizers, post-contrast scans were obtained with a time gap of 15 min post-contrast administration. A total number of 680 myocardial segments and corresponding 120 vascular territories were assessed. MRI and ${ }^{18}$ F-FDG PET images were analyzed using a 17-segment model as proposed by AHA. Sensitivity, specificity, positive predictive value, and negative predictive value of non-breath-hold rapid delayed contrast-enhanced cardiac MR imaging protocol for assessing myocardial viability (on segment analysis) was 95.5\%, 65.59\%, $88.0 \%$, and $84.72 \%$, respectively, and of vascular territory analysis were $96.77 \%, 92.59 \%, 97.83 \%$, and $89.29 \%$, respectively, in relation to ${ }^{18} \mathrm{~F}$-FDG PET used as the reference standard. Spearman's rank correlation coefficient is 0.62 .
\end{abstract}

Conclusion: This modified non-breath-hold delayed contrast-enhanced cardiac MR imaging protocol is a reliable tool to answer the clinically relevant question of myocardial viability with a significant reduction in acquisition time and overcomes the limiting need of breath hold.

Keywords: Myocardial viability, Magnetic resonance imaging, Positron emission tomography

\footnotetext{
* Correspondence: rohitaggy@gmail.com

'Department of Radiology, 7 Air Force Hospital, Nathu Singh Road, Kanpur

Cantonment, Kanpur, Uttar Pradesh, India

Full list of author information is available at the end of the article
}

Springer Open (c) The Author(s). 2020 Open Access This article is licensed under a Creative Commons Attribution 4.0 International License, which permits use, sharing, adaptation, distribution and reproduction in any medium or format, as long as you give appropriate credit to the original author(s) and the source, provide a link to the Creative Commons licence, and indicate if changes were made. The images or other third party material in this article are included in the article's Creative Commons licence, unless indicated otherwise in a credit line to the material. If material is not included in the article's Creative Commons licence and your intended use is not permitted by statutory regulation or exceeds the permitted use, you will need to obtain permission directly from the copyright holder. To view a copy of this licence, visit http://creativecommons.org/licenses/by/4.0/. 


\section{Background}

Ischemic heart disease (IHD) remains the principal cause of mortality and morbidity worldwide [1]. Half a century ago, observations of reversibility of left ventricular dysfunction in patients with IHD came into being with revascularization procedures like invasive percutaneous coronary angioplasty and coronary artery bypass graft [2]. However, not all patients are likely to benefit from the revascularization procedures. On echocardiography, the hypokinetic or akinetic myocardium was deemed as pathological. This pathological myocardium could be stunned and hibernating myocardium or it could be fibrosed myocardium. The stunned and hibernating are the terms that are used to describe the myocardium, which is viable. However, due to reduced perfusion, it is hypokinetic or akinetic. The stunned and hibernating myocardia are likely to recover function after successful revascularization. If the pathological myocardium is infarcted, it is unlikely to recover after revascularization. Hence, for clinical decision making, it is extremely important to delineate viable areas from non-viable areas in dysfunctional myocardium with high accuracy as only the former is likely to recover its contractile function on successful revascularization [3]. Delayed contrastenhanced cardiac magnetic resonance imaging (DCECMR) is a well-established magnetic resonance imaging (MRI) technique for evaluation of myocardial viability [4]. This technique prognosticates myocardial viability with high accuracy and spatial resolution. However, the routine use of DCE-CMR for myocardial viability is limited by a multitude of factors [5] primarily being the inability of patients to hold breath and lengthy acquisition time which can impose a significant discomfort to patients. This increased gantry time is also administratively difficult in multimodality centers without a dedicated cardiac MRI scanner. The important question to which the referring cardiologists/cardiac surgeons are seeking answer is that "What are the viable segments that will benefit from revascularization?" In this study, we have analyzed the utility of a non-breath-hold DCE-CMR imaging protocol for myocardial viability assessment. This protocol mitigates the requirement of breath hold sequences and significantly reduces acquisition time while retaining the diagnostic potential of answering the critical question.

We have evaluated myocardial viability assessment by the proposed modified DCE-CMR protocol as compared to viable tissue defined by ${ }^{18}$ flourodeoxy glucose positron emission tomography $\left({ }^{18} \mathrm{~F}\right.$-FDG PET) in patients with IHD [6] as the nuclear imaging technique has been well validated as a reliable marker of myocardial viability.

\section{Methods}

A total of 52 consecutive patients of IHD with reduced left ventricle ejection fraction (as assessed on echocardiography) and diagnosed with triple vessel disease with or without chronic total occlusion on catheter angiography were considered for revascularization surgery and were referred for viability assessment by ${ }^{18}$ F-FDG PET. All these cases referred for ${ }^{18}$ F-FDG PET were included in our study. Patients with history of myocardial infarction within 6 weeks before ${ }^{18} \mathrm{~F}$-FDG PET/MRI, those with contraindications to ${ }^{18} \mathrm{~F}$-FDG PET/MRI, and those with unstable angina and advanced heart failure (New York Heart Association Class IV) were excluded from the study. The protocol was approved by the institutional ethics committee. Written informed consent was obtained from patients.

A total of 7 patients were excluded from the study due to various causes including non-compatible metallic implant $(n=02)$, deranged renal function $(n=02)$, and uncontrolled diabetes not suitable for ${ }^{18} \mathrm{~F}$-FDG $\operatorname{PET}(n=3)$. All the patients underwent DCE-CMR and ${ }^{18} \mathrm{~F}-\mathrm{FDG}$ PET within a gap of $24-48 \mathrm{~h}$ of each other.

DCE-MR studies were performed using a 1.5 Tesla MRI scanner (Magnetom Avanto, Siemens Erlangen, Germany). To obtain the correct inclinations for imaging in the cardiac axis, localizers were acquired in short-axis (SA), vertical long-axis (VLA), and horizontal long-axis (HLA) planes [7]. Bolus injection of $0.2 \mathrm{mmol} / \mathrm{kg}$ body weight gadolinium was administered. After a gap of $12-$ 14 min, myocardial nulling time was chosen. Late gadolinium enhancement (LGE) was studied using phasesensitive inversion recovery sequence (matrix $192 \times 144$, voxel size $2.4 \times 1.8 \times 6 \mathrm{~mm}$, time to echo $-1.08 \mathrm{~ms}$, time to resonance $-700 \mathrm{~ms}$, flip angle $50^{\circ}$, and the bandwidth $1180 \mathrm{~Hz} /$ pixel). Images were obtained in SA, VLA, and HLA planes. All these MRI sequences were obtained in free breathing.

All patients were studied with ${ }^{18}$ F-FDG PET on a 64 slice Time of Flight Hybrid PET/CT scanner (Discovery 90, GE Healthcare Waukesha, Wisconsin, USA). Patients were imaged in postprandial state receiving glucose and insulin (if required) before and during procedure, according to a standardized protocol. Computed tomography $(\mathrm{CT})$ data was used for attenuation correction. PET data was acquired in a three-dimensional mode, and data realigned to generate short- and long-axis views for visual analysis. Image analysis was done by both observers independently for MRI and PET images respectively. Only in cases where there was a discrepancy both observers reviewed the images together and only after consensus findings were included in results.

Forty cases were included in the final analysis as 05 patients could not complete MRI due to claustrophobia. MRI as well as ${ }^{18}$ F-FDG PET images were analyzed 
using 17 Segment model as proposed by the American Heart Association [8]. The results were also evaluated vascular territory wise. The vascular territories evaluated were LAD (left anterior descending), LCx (left circumflex artery), and RCA (right coronary artery) territory in each patient. The extent of LGE was divided into transmural (>50\% LGE) and subendocardial (<50\%LGE). Transmural LGE was considered as non-viable, and subendocardial LGE was considered as viable myocardium [9].

${ }^{18}$ F-FDG PET data was analyzed for FDG uptake both subjectively by visual analysis and objectively by automated semiquantitative image analyzer on a polar plot which divided the heart into 17 segments, and viable myocardium in each segment was depicted as a percentage. The segments were classified into 2 categories-uptake less than 50\% (non-viable) and uptake more than 50\% (viable) [10]. For both MRI and ${ }^{18}$ F-FDG PET, percentage viability of segments and coronary territories were recorded. Using PET as the reference standard, specificity, sensitivity, positive likelihood ratio, negative likelihood ratio, positive predictive value, negative predictive value, and accuracy were calculated for non-breath-hold cardiac DCE-CMR. The percentage viability in a given vascular territory or arterial segment on both modalities were correlated using Rho correlation coefficient.

\section{Results}

Of 52 consecutive patients who were referred for myocardial viability assessment, 12 patients were excluded from the study due to various reasons as mentioned above. A total of 40 patients with 120 coronary territories and 680 myocardial segments were analyzed. Ninety percent $(n=36)$ were males; the mean age of the study subjects was 54.5 years ranging from 21 to 80 years.

On DCE-CMR, 536 (80\%) myocardial segments showed <50\% LGE and 144 (20\%) segments showed > 50\% LGE. On PET, 494 (72.64\%) segments were viable and 186 (27.36\%) segments were non-viable. Sensitivity, specificity, and accuracy of DCE-CMR for predicting viability of myocardial segments were $95.5 \%, 65.59 \%$, and $87.35 \%$, respectively (Table 1 )

Coronary territory wise viability analysis showed less than 50\% LGE in 27 LAD, 28 RCA, and 37 LCx territories on DCE-MRI as compared to 28 LAD, 27 RCA, and $34 \mathrm{LCx}$ viable territories on PET. Sensitivity, specificity, and accuracy of DCE-CMR for predicting viability of coronary vascular territories were $96.77 \%, 92.59 \%$, and $95.83 \%$, respectively (Table 2). Rho correlation coefficient was calculated to be 0.62 for segmental analysis and 0.80 for coronary territorial analysis. This denotes moderate to strong agreement between DCE-CMR using free-breathing protocol and ${ }^{18} \mathrm{~F}$-FDG PET for viability assessment.

\section{Discussion}

Myocardial viability is a vital clinical concern in a patient of ischemic heart disease prior to any revascularization procedure. Among various modalities used for viability assessment, DCE-CMRI has been proven to be extremely accurate. The conventional cardiac viability protocol can last for 60-90 min and consists of localizers followed by dark blood and bright blood imaging for the 3 orthogonal views and the cine breath hold steady state free precision sequence for assessing wall motion, enddiastolic wall thickness, and ejection fraction. This is followed by gadolinium administration, TI scout, and phase-sensitive inversion recovery images in SA, VLA, and HLA views [11].

Although cardiac MRI is a versatile and highly accurate imaging modality, there are several practical limitations to the use of the technique. Dickstein (2008) mentioned that compared with $\mathrm{CT}$ scans and cardiac ultrasound, cardiac MRI examinations are timeconsuming and prolonged examination durations can be expected as most routine cardiac MRI techniques are

Table 1 Myocardial segment wise viability analysis

\begin{tabular}{|c|c|c|}
\hline & Viable on PET & Non-viable on PET \\
\hline LGE $<50 \%$ & 472 (true positive) & 64 (false positive) \\
\hline \multirow[t]{2}{*}{ LGE $>50 \%$} & 22 (false negative) & 122 (true negative) \\
\hline & Statistical analysis & \\
\hline Parameter & Value & 95\% Confidence interva \\
\hline Sensitivity & $95.55 \%$ & $93.33-97.19 \%$ \\
\hline Specificity & $65.59 \%$ & $58.29-72.39 \%$ \\
\hline Positive likelihood ratio & 2.78 & $2.27-3.39$ \\
\hline Negative likelihood ratio & 0.07 & $0.04-0.10$ \\
\hline Positive predictive value & $88.06 \%$ & $85.8-90 \%$ \\
\hline Negative predictive value & $84.72 \%$ & $74.44-89.42 \%$ \\
\hline Accuracy & $87.35 \%$ & $84.62-89.76 \%$ \\
\hline
\end{tabular}


Table $\mathbf{2}$ Coronary arterial territory wise viability analysis

\begin{tabular}{lll}
\hline & Viable on PET & Non-viable on PET \\
\hline LGE $<50 \%$ & 90 (true positive) & 2 (false positive) \\
LGE $>50 \%$ & 3 (false negative) & 25 (true negative) \\
Statistical analysis & & \\
Parameter & Value & $95 \%$ Confidence interval \\
Sensitivity & $96.77 \%$ & $90.86-99.33 \%$ \\
Specificity & $92.59 \%$ & $75.71-99.09 \%$ \\
Positive likelihood ratio & 13.06 & $3.44-49.60$ \\
Negative likelihood ratio & 0.03 & $0.01-0.11$ \\
Positive predictive value & $97.83 \%$ & $92.22-99.42 \%$ \\
Negative predictive value & $89.29 \%$ & $73.14-96.23 \%$ \\
Accuracy & $95.83 \%$ & $90.54-98.63 \%$ \\
\hline
\end{tabular}

dependent upon image acquisition during breath hold to eliminate movement artifacts [12]. Both image quality and the duration of the examination are highly dependent upon the patient's ability to do repeated performance of breath hold of 5-10 s. In patients who are unable to perform breath hold correctly, a poor image quality must be anticipated that can be proven difficult for satisfactory interpretation of images. Hence, in this study, the proposed non-breath-hold rapid cardiac MRI protocol was used. A free-breathing protocol precludes the use of cine imaging. These cine sequences provide vital information about regional wall motion abnormalities apart from assessment of cardiac function and enddiastolic wall thickness. Presence of LGE in an area of regional wall motion abnormality denotes non-viable myocardium. Recent evidence suggests that late gadolinium enhancement may be independent of regional wall motion abnormality in predicting myocardial viability [9].

The two most important predictors for predicting recovery of the ventricular function after revascularization procedures are the extent of infarct as assessed by LGE and end-diastolic wall thickness (EDWT) which can be assessed by the images in SA view in bright blood imaging sequence. However, Krittayaphong et al. in 2008 compared the value of LGE and EDWT assessed by cardiovascular magnetic resonance in predicting recovery of left ventricular function after coronary artery bypass graft surgery (CABG). The study concluded that LGE and EDWT are independent predictors for functional recovery after revascularization. However, LGE appears to be a more important factor and independent of EDWT [13]. Various studies have also postulated that delayed gadolinium enhancement can effectively demonstrate the extent of infarct and the long-term prognosis as well as the incidence of adverse events after revascularization. A study by Lee et al. in 2016 evaluated the impact of delayed gadolinium enhancement on the long-term progress in patients undergoing $\mathrm{CABG}$ and concluded that the extent of delayed contrast enhancement is a strong predictor of adverse cardiac events independent of left ventricular function [14]. In 2018, Lim et al. also concluded that the extent of myocardial viability as assessed by cardiac magnetic resonance late gadolinium enhancement appears to identify patients with a differential survival benefit from CABG [15]. In a study by Gerber (2012), it was concluded that the presence of dysfunctional viable myocardium by delayed contrast enhancement is an independent predictor of mortality in patients with ischemic LV dysfunction [16]. We studied feasibility of non-breath-hold abridged viability protocol to predict myocardial viability using late gadolinium enhancement as a sole marker for viability. Estimation of glucose metabolism by ${ }^{18} \mathrm{~F}$-FDG PET has been reported to accurately differentiate between viable and non-viable myocardial tissue in many studies $[17,18]$ and, thus, was used as a reference method.

Out of the total evaluated 120 coronary arterial territories on DCE-CMR (3 vascular territories per patient): 15 patients had non-viable LAD territory, 13 patients had non-viable RCA territory, and 7 patients had nonviable LCX territory. When the results of the vascular territories were compared between DCE-CMR and ${ }^{18} \mathrm{~F}$ FDG PET only 5 out of 120 vascular territories show discrepant results. Fifteen patients showed non-viable myocardium on DCE-CMR. Out of these, 14 patients were showing similar findings in ${ }^{18}$ F-FDG PET (Figs. 1 and 2). The results of this study demonstrate a moderate to strong agreement between DCE-MRI and ${ }^{18}$ F-FDG PET (Figs. 3 and 4) Agreement between two modalities was obtained in 595 myocardial segments (88.5\%), resulting in a rho value of 0.62 .

In our study, free-breathing DCE-CMR protocol is seen to be highly sensitive and accurate on a per coronary arterial territory basis. These results are comparable with the results of $\mathrm{Li}$ et al. who have compared the use of late gadolinium enhancement on breath hold cardiac MRI with a ${ }^{18}$ F-FDG PET scan [18].

Segment to segment analysis for discordant results was done by a nuclear medicine specialist ( 12 years' experience) and radiologist trained in cross-sectional imaging (11 years' experience), and the following reasons were attributed to for the discrepancy on segment to segment analysis.

MRI can delineate segments more accurately, because the border between enhanced and normal areas is distinct, whereas in nongated ${ }^{18} \mathrm{~F}$-FDG PET images, the border between normal and defect areas is less well defined. Moreover, for the semiquantitative analysis of the segments, polar plots were used which tend to average out the segments and hence give erroneous readings at the border segments. All comparative studies have the 


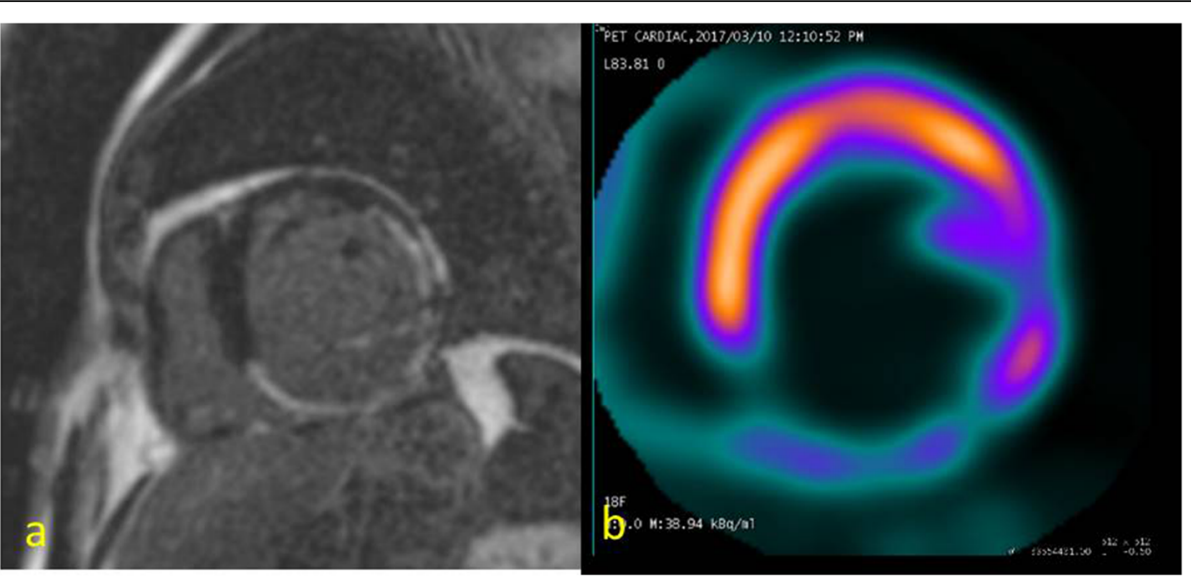

Fig. 1 A 64-year-old male with triple vessel disease. a Free-breathing delayed contrast-enhanced MRI in short-axis view reveals late gadolinium enhancement in $<50 \%$ myocardial thickness suggestive of subendocardial infarct in left circumflex and late gadolinium enhancement in complete thickness of right coronary artery territory suggestive of transmural infarct. $\mathbf{b}^{18} \mathrm{~F}$-FDG PET reveals normal uptake in left anterior descending artery $<50 \%$ uptake in the right coronary artery territory and $>50 \%$ uptake in the left circumflex territory

potential for anatomical misalignment. Although shortaxis views were used in both modalities, the visualization of the inferior interception of the ventricles is especially difficult in patients with extensive scar tissue, because regions are not always clearly definable in ${ }^{18}$ F-FDG PET. Hence, there is a difference in specificity when comparing individual segments and coronary territories.

Assessment of wall thickness is limited by the spatial resolution of nongated ${ }^{18}$ F-FDG PET, and epicardial tracer activity may mask small subendocardial defects. Therefore, MRI, with its better spatial resolution provides excellent delineation of scar tissue as compared to ${ }^{18}$ F-FDG PET. FDG is a marker for viability, whereas delayed contrast enhancement is considered a marker for scar tissue. Thus, a relatively small number of viable cells may show increased FDG uptake, indicating viability, whereas structural changes may already coexist and alter gadolinium kinetics. Therefore, ${ }^{18}$ F-FDG PET may show viability in segments with LGE, depending on the relative contribution of viable and fibrotic tissue. Third, an increase in regional signal intensity (due to gadolinium) may be easier to interpret than a regional comparison of flow and metabolism by PET. Authors postulate these reasons to explain the occurrence of three false negative cases (in coronary territory wise analysis) in our study. A high intracellular glucose level is unlikely to make the cells hungry for glucose when tracer is injected; hence, these cases are not likely to show any uptake of tracer despite the territory being viable. This mechanism could possibly explain the occurrence of two false positive cases (in coronary territory wise analysis) in our study. The traditional method used

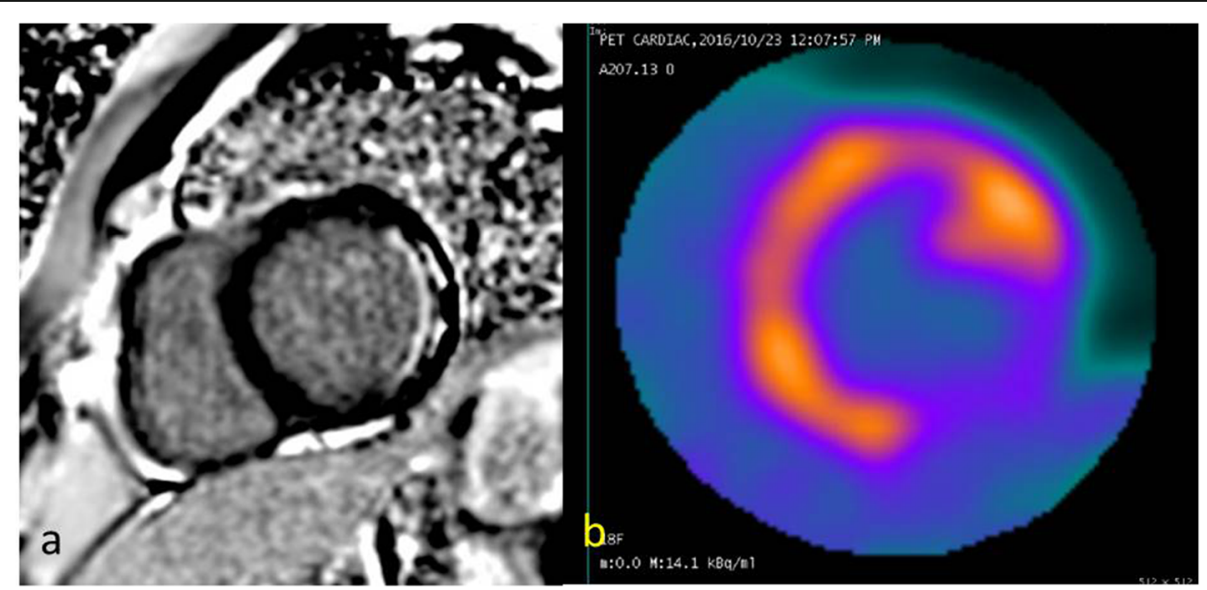

Fig. 2 A 60-year-old male with triple vessel disease. a Free-breathing delayed contrast-enhanced MRI in short-axis view reveals late gadolinium enhancement in $<50 \%$ myocardial thickness suggestive of subendocardial infarct in the left circumflex artery territory. $\mathbf{b}{ }^{18} \mathrm{~F}-\mathrm{FDG}$ PET reveals normal uptake in the left anterior descending artery and right coronary artery territory and reduced uptake in left circumflex territory 


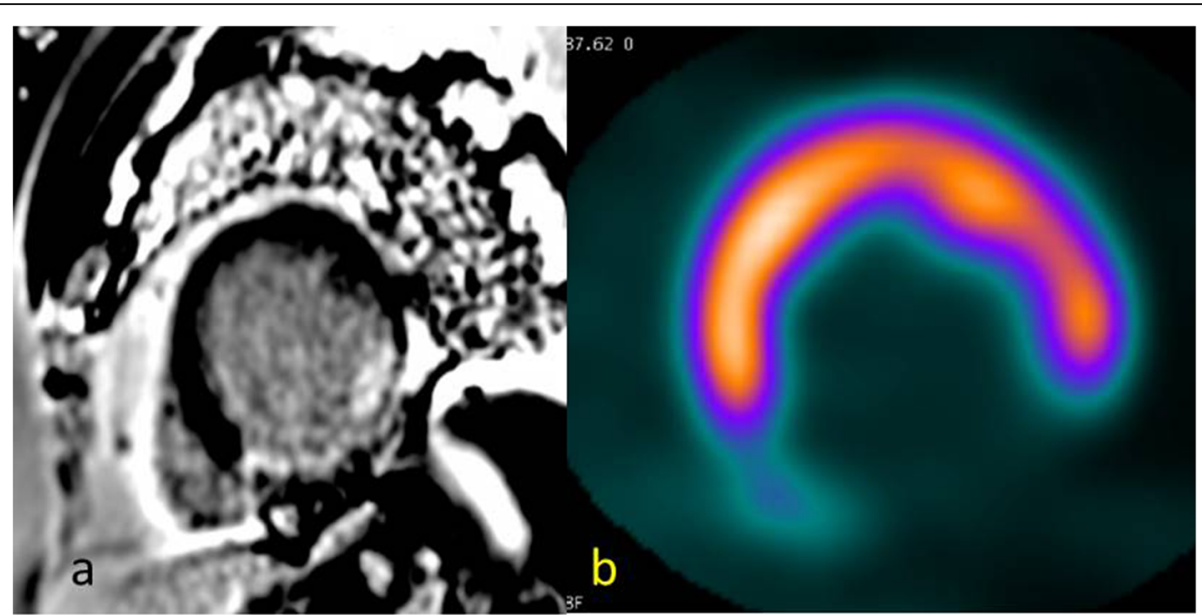

Fig. 3 A 57-year-old male with triple vessel disease. a Free-breathing delayed contrast-enhanced MRI in short-axis view reveals late gadolinium enhancement in $>50 \%$ myocardial thickness suggestive of transmural infarct in the right coronary artery territory suggestive of transmural infarct. b ${ }^{18} \mathrm{~F}$-FDG PET reveals no uptake in the right coronary artery territory suggestive of non-viable segment

in viability imaging includes presence of regional wall motion abnormality in the segment showing delayed contrast enhancement. We have not done routine breath hold cine sequences, and thus, the study does not have data about regional wall motion abnormalities. Despite this, our results have shown that LGE alone (even when done in free breathing) can answer the clinically pertinent question about presence or absence of viable myocardium so as to decide the further line of treatment.

As per the authors' knowledge, no other study, using a completely free-breathing protocol exists. In the present study, the short acquisition times and high spatial resolution of the DCE-CMR allowed to perform the cardiac examination in a short time with high definition of myocardial scarring. DCE-CMR as a technique stands on its own with comparable sensitivity and specificity with ${ }^{18}$ F-FDG PET and better morphological and functional delineation of the myocardium. Cardiac MRI has following additional advantages in assessment of myocardial viability in patients of IHD:

- ${ }^{18}$ F-FDG PET requires elaborate patient preparation unlike DCE-CMR

- There is no radiation exposure.

- Better availability of MRI scanners as compared to PET scanners.

- Differentiation of ischemic and non-ischemic cardiomyopathies.

- Cheaper alternative to ${ }^{18}$ F-FDG PET.

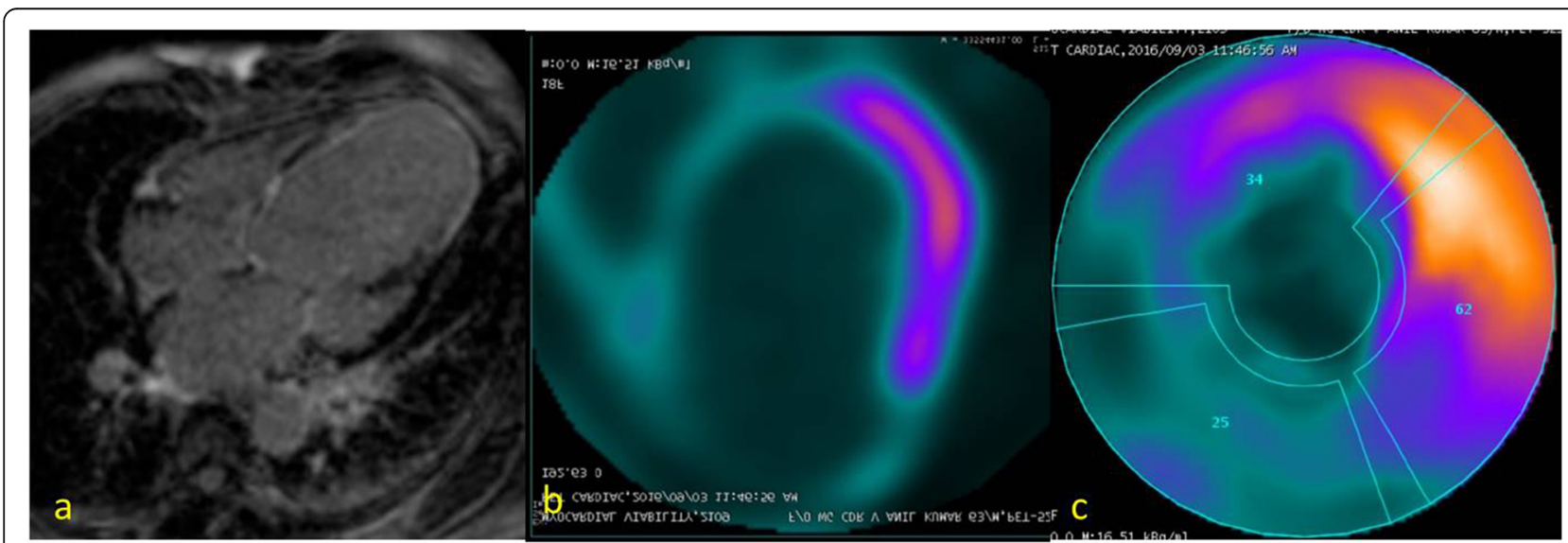

Fig. 4 A 57-year-old male with triple vessel disease. a Free-breathing delayed contrast-enhanced MRI in horizontal long-axis view reveals late gadolinium enhancement in $>50 \%$ myocardial thickness at the septum, apex and inferior wall of left ventricle suggestive of transmural infarct in left anterior descending and right coronary artery territory. $\mathbf{b}{ }^{18} \mathrm{~F}$-FDG PET reveals non-viable left anterior descending and right coronary artery territory. c Polar plot in the same patient reveals non-viable left anterior descending and right coronary artery territory 
The proposed modified DCE-CMR free-breathing protocol offers the advantages of markedly reduced scan time $(20-25 \mathrm{~min})$, better acceptability by patients, and efficient management of gantry time.

\section{Study limitations}

Cine sequences provide additional information about myocardial function, chamber volumes, and regional wall motion abnormalities. Since we did not acquire cine sequence, this data is not available in our study. A direct comparison of our free-breathing protocol with conventional breath hold cardiac MRI protocol in the same patient would have been a more accurate way of assessing the efficacy of proposed modification. However, the same could not be done due to varying reasons like limited gantry time.

\section{Conclusion}

Non-breath-hold DCE-CMR with significant reduction in acquisition time is a reliable tool for myocardial viability assessment to answer the clinically relevant question of myocardial viability. Wider public access to MRI scanners, relatively lower cost, and eliminating the use of ionizing radiation could definitely make this modified cardiac MRI protocol a practically viable alternative to the current gold standard ${ }^{18}$ F-FDG PET. Radiologists and MRI technicians need to specifically familiarize themselves with cardiac imaging protocols and to manage protocol modifications to retain continued clinical acceptability.

\section{Abbreviations}

DCE-CMR: Delayed contrast-enhanced cardiac magnetic resonance imaging; MRI: Magnetic resonance imaging; IHD: Ischemic heart disease; MI: Myocardial infarction; ${ }^{18}$ F-FDG PET: ${ }^{18}$ Flourodeoxy glucose positron emission tomography; SA: Short axis; VLA: Vertical long axis; HLA: Horizontal long axis; CT: Computed tomography; LAD: Left anterior descending; LCX: Left circumflex artery; RCA: Right coronary artery; DCE: Delayed contrast enhanced; LGE: Late gadolinium enhancement; EDWT: End-diastolic wall thickness; CABG: Coronary artery bypass graft surgery

\section{Acknowledgments}

Nil

\begin{abstract}
Authors' contributions
All authors certify that they have made substantive and intellectual contribution to the article and assume public responsibility for its content. It is confirmed that all authors have read and approved the manuscript for submission. The individual contributions to the manuscript of each author are as follows: RA did the study conception and design of the work and acquisition, analysis, and interpretation of the data and drafted the work. GS did the acquisition, analysis, interpretation of the data and drafted the work. HS did the study conception and design of the work, and acquisition and analysis. RAG substantively revised the manuscript and approved the submitted version. IM substantively revised the manuscript and approved the submitted version.
\end{abstract}

\section{Funding}

No separate funding was required for this study

\section{Availability of data and materials}

The datasets used and/or analyzed during the current study are available from the corresponding author on reasonable request

Ethics approval and consent to participate

Institutional ethical committee approval was obtained. Written informed consent was obtained from participants on informed consent proforma.

Consent for publication

Written informed consent was obtained form study participants.

\section{Competing interests}

The authors declare that they have no competing interests

\section{Author details}

'Department of Radiology, 7 Air Force Hospital, Nathu Singh Road, Kanpur Cantonment, Kanpur, Uttar Pradesh, India. ${ }^{2}$ Department of Radiology, Command Hospital Air Force, Bangalore, India. ${ }^{3}$ Commandant, Military Hospital Roorkee, Uttarakhand, India. ${ }^{4}$ Department of Obstetrics and Gynaecology, Armed Forces Medical College, Pune, Maharashtra, India.

Received: 29 April 2020 Accepted: 17 August 2020

Published online: 01 September 2020

References

1. Virani S, Alonso A, Benjamin E et al (2020) Heart Disease and Stroke Statistics-2020 Update: a report from the American Heart Association. Circulation. https://doi.org/10.1161/cir.0000000000000757

2. Velazquez $E$, Lee $K$, Jones $R$ et al (2016) Coronary-artery bypass surgery in patients with ischemic cardiomyopathy. N Engl J Med 374:1511-1520. https://doi.org/10.1056/nejmoa1602001

3. Allman K, Shaw L, Hachamovitch R, Udelson J (2002) Myocardial viability testing and impact of revascularization on prognosis in patients with coronary artery disease and left ventricular dysfunction: a metaanalysis. J Am Coll Cardiol 39:1151-1158. https://doi.org/10.1016/s07351097(02)01726-6

4. Jimenez Juan L, Crean A, Wintersperger B (2015) Late gadolinium enhancement imaging in assessment of myocardial viability. Radiol Clin N Am 53:397-411. https://doi.org/10.1016/j.rcl.2014.11.004

5. Löffler A, Kramer C (2018) Myocardial viability testing to guide coronary revascularization. Interven Cardiol Clin 7:355-365. https://doi.org/10.1016/j. iccl.2018.03.005

6. Shabana A, El-Menyar A (2012) Myocardial viability: what we knew and what is new. Cardiol Res Pract 2012:1-13. https://doi.org/10.1155/2012/ 607486

7. Burbank F, Parish D, Wexler L (1988) Echocardiographic-like angled views of the heart by MR imaging. J Comput Assist Tomogr 12:181-195. https://doi. org/10.1097/00004728-198803000-00001

8. Cerqueira M, Weissman N, Dilsizian V et al (2002) Standardized myocardial segmentation and nomenclature for tomographic imaging of the heart. Circulation 105:539-542. https://doi.org/10.1161/hc0402.102975

9. Souto A, Souto R, Teixeira I, Nacif M (2017) Myocardial viability on cardiac magnetic resonance. Arq Bras Cardiol. https://doi.org/10.5935/abc.20170056

10. Slart R, Bax J, Veldhuisen $D$ et al (2006) Prediction of functional recovery after revascularization in patients with coronary artery disease and left ventricular dysfunction by gated FDG-PET. J Nucl Cardiol 13:210-219. https://doi.org/10.1007/bf02971245

11. Kramer C, Barkhausen J, Flamm S et al (2013) Standardized cardiovascular magnetic resonance (CMR) protocols 2013 update. J Cardiovasc Magn Reson. https://doi.org/10.1186/1532-429x-15-91

12. Dickstein K (2008) Clinical utilities of cardiac MRI. e-Journal of Cardiology Practice 6:39.

13. Krittayaphong R, Laksanabunsong P, Maneesai A et al (2008) Comparison of cardiovascular magnetic resonance of late gadolinium enhancement and diastolic wall thickness to predict recovery of left ventricular function after coronary artery bypass surgery. J Cardiovasc Magn Reson. https://doi.org/10. 1186/1532-429x-10-41

14. Lee S, Yoon Y, Kim J et al (2016) Long-term prognostic value of late gadolinium-enhanced magnetic resonance imaging in patients with and without left ventricular dysfunction undergoing coronary artery bypass 
grafting. Am J Cardiol 118:1647-1654. https://doi.org/10.1016/j.amjcard.2016. 08.043

15. Lim F, Yap J, Gao F et al (2018) Impact of myocardial viability assessed by cardiac magnetic resonance on survival in patients with severe ischemic left ventricular dysfunction. J Am Coll Cardiol 71:A1641. https://doi.org/10.1016/ s0735-1097(18)32182-x

16. Gerber B, Rousseau M, Ahn S et al (2012) Prognostic value of myocardial viability by delayed-enhanced magnetic resonance in patients with coronary artery disease and low ejection fraction. J Am Coll Cardiol 59:825835. https://doi.org/10.1016/j.jacc.2011.09.073

17. Matsunari I, Taki J, Nakajima K et al (2003) Myocardial viability assessment using nuclear imaging. Ann Nucl Med 17:169-179. https://doi.org/10.1007/ bf02990019

18. Li J, He Y, Dong W et al (2019) Comparison of cardiac MRI with PET for assessment of myocardial viability in patients with coronary chronic total occlusion. Clin Radiol 74:410.e1-410.e9. https://doi.org/10.1016/j.crad.2019. 01.021

\section{Publisher's Note}

Springer Nature remains neutral with regard to jurisdictional claims in published maps and institutional affiliations.

\section{Submit your manuscript to a SpringerOpen ${ }^{\odot}$ journal and benefit from:}

- Convenient online submission

- Rigorous peer review

- Open access: articles freely available online

High visibility within the field

- Retaining the copyright to your article

Submit your next manuscript at $\boldsymbol{\nabla}$ springeropen.com 